\title{
Gastrointestinal bleeding from jejunal heterotopic pancreas diagnosed by deep small-bowel enteroscopy
}

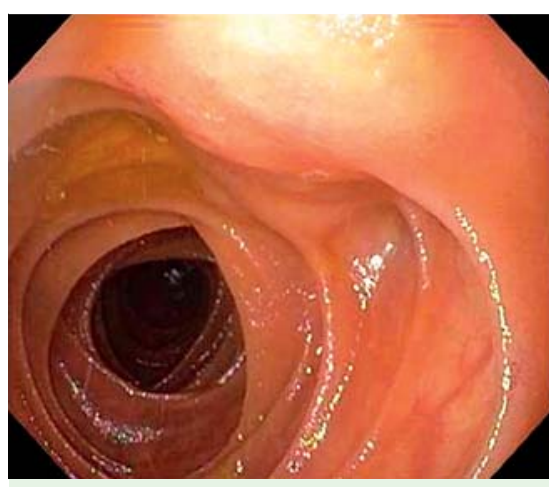

Fig. 1 View during spiral enteroscopy showing a medium-sized subepithelial mass with mucosal nodularity in the distal jejunum.

A 70-year-old man presented with a 2-week history of overt (melena) and obscure gastrointestinal bleeding requiring an 8-unit blood transfusion. He underwent upper gastrointestinal endoscopy, colonoscopy, and push enteroscopy, all of which were nondiagnostic. A video capsule endoscopy, which reached the cecum at 6 hours and 21 minutes, showed active bleeding at 2 hours and 18 minutes without an obvious underlying lesion.

The patient was transferred to our facility and underwent antegrade deep (spiral) enteroscopy. A medium-sized subepithelial mass with mucosal nodularity was found $90 \mathrm{~cm}$ distal to the ligament of Treitz ( Fig. 1). Standard forceps biopsies were diagnostic of heterotopic pancreas. At laparoscopy, the tumor was easily identified at the site of the endoscopically placed tattoo ( Fig.2) and jejunal resection was subsequently performed. Pathologic evaluation of the surgical specimen confirmed heterotopic pancreas ( Fig. 3a) with focal areas of mucosal ulceration ( Fig.3b). Postoperatively, the patient did well and he had no recurrent bleeding at his 1-month follow-up visit.

Heterotopic (ectopic) pancreas is a relatively rare submucosal tumor that occurs most commonly in the stomach and duodenum [1]. Most tumors are discovered incidentally during radiographic or endoscopic examination, but a small proportion of patients with heterotopic pancreas can present with symptoms such as abdominal pain or bleeding [2]. A few recent

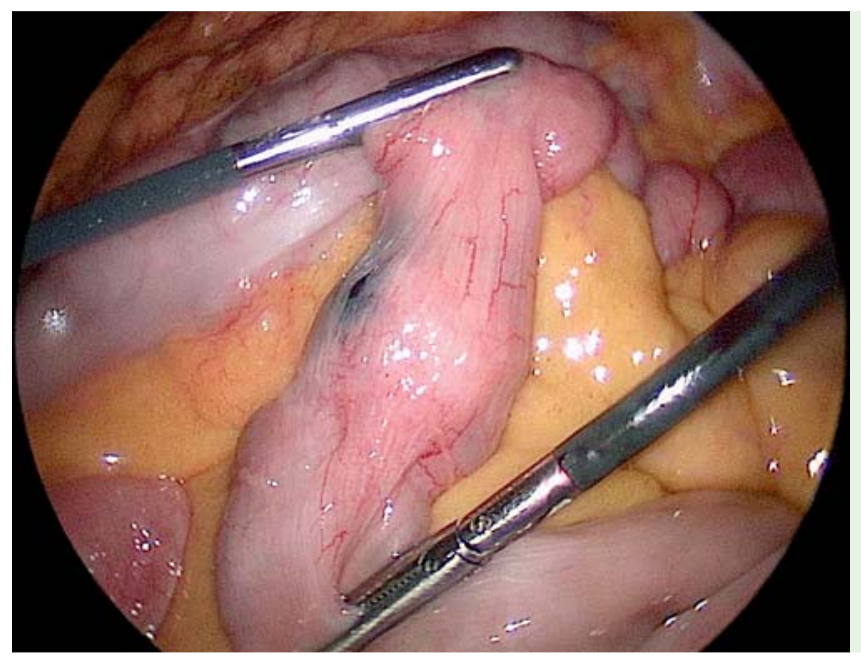

Fig. 2 Laparoscopic view of the jejunal mass with the adjacent tattoo mark clearly visible.
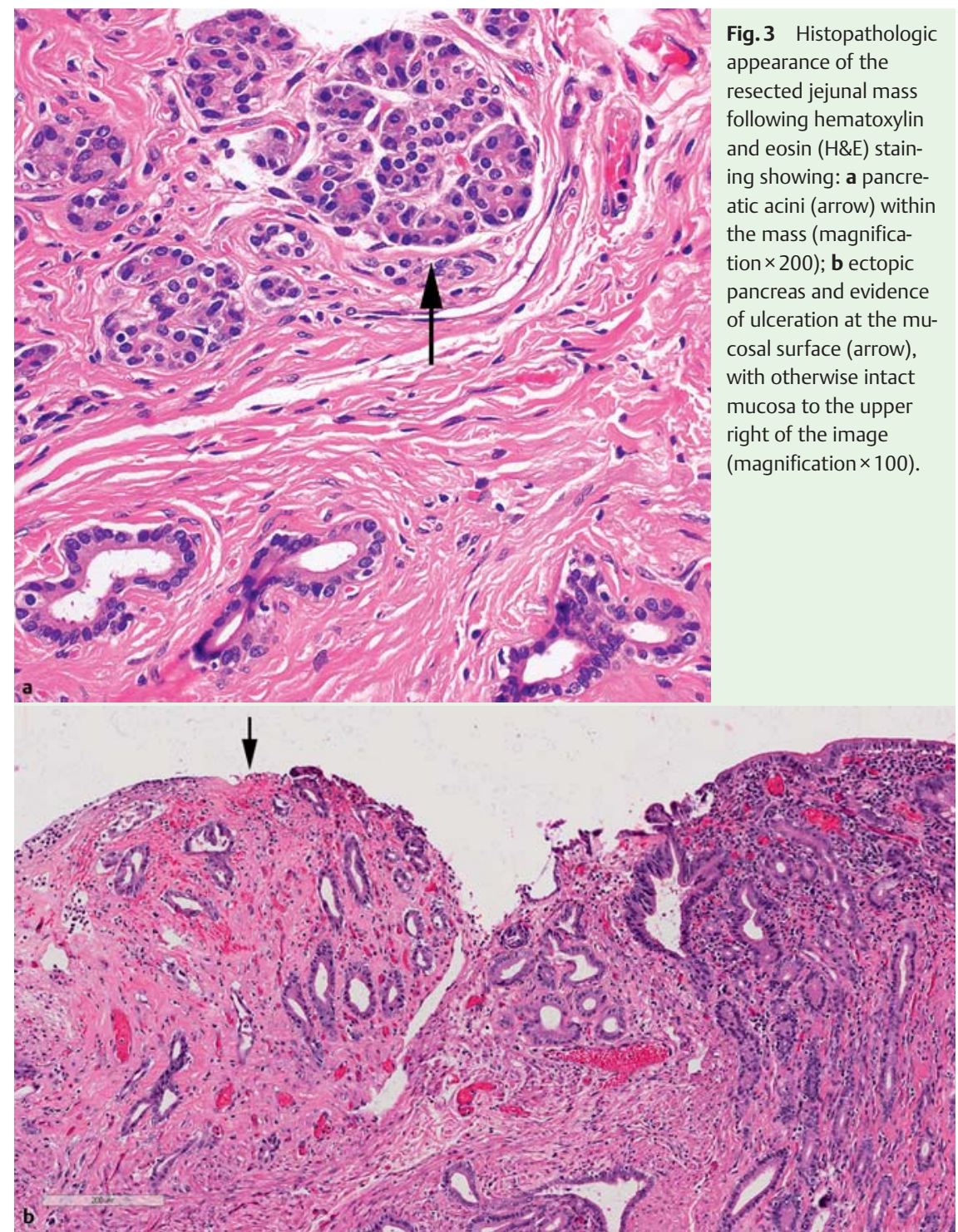
case reports have described the diagnosis of small-bowel heterotopic pancreas by capsule or balloon enteroscopy [3-5], but to our knowledge, this is the first reported case of heterotopic pancreas diagnosed by spiral enteroscopy.

Newer tools for evaluating the small intestine have expanded the diagnostic capabilities, but laparoscopic resection remains the definitive treatment for symptomatic heterotopic pancreas. Small-bowel heterotopic pancreas should be considered in the differential diagnosis of obscure gastrointestinal bleeding. Deep small-bowel enteroscopy can identify the lesion, provide tissue diagnosis, and allow placement of a tattoo mark for easy localization at the time of laparoscopy.

Endoscopy_UCTN_Code_CCL_1AC_2AH

Competing interests: None

\section{J. Blair Williamson¹, David H. Gonzalo², Juan F. Alvarez ${ }^{3}$, Georgios Rossidis ${ }^{3}$, Peter V. Draganov ${ }^{1}$}

${ }^{1}$ Department of Medicine, University of Florida, Gainesville, Florida, USA

${ }^{2}$ Department of Pathology, University of Florida, Gainesville, Florida, USA

${ }^{3}$ Department of Surgery, University of Florida, Gainesville, Florida, USA

\section{References}

1 Dolan RV, ReMine WH, Dockerty MB. The fate of heterotopic pancreatic tissue. A study of 212 cases. Arch Surg 1974; 109: 762-765

2 Hsia CY, Wu CW, Lui WY. Heterotopic pancreas: a difficult diagnosis. J Clin Gastroenterol 1999; 28: $144-147$

3 Lee MJ, Chang JH, Maeng IH et al. Ectopic pancreas bleeding in the jejunum revealed by capsule endoscopy. Clin Endosc 2012; 45: $194-1974$

4 Tsurumaru D, Utsunomiya T, Kayashima K et al. Heterotopic pancreas of the jejunum diagnosed by double-balloon enteroscopy. Gastrointest Endosc 2007; 66: 1026-1027

5 Takeda Y, Nakase H, Chiba T. Ectopic pancreas at the jejunum. Dig Liver Dis 2011; 43: e6
Bibliography

Dol http://dx.doi.org/

10.1055/s-0034-1365747

Endoscopy 2014; 46: E297-E298

(c) Georg Thieme Verlag KG

Stuttgart · New York

ISSN 0013-726X

\section{Corresponding author}

\section{J. Blair Williamson, MD}

University of Florida

Division of Gastroenterology, Hepatology,

and Nutrition

1329 SW 16th

Ave, Suite 5251

PO Box 100214

Gainesville, FL 32608

USA

Fax: +1-352-392-3618

blair.williamson@medicine.ufl.edu 